\title{
Chronic Encapsulated Intracerebral Hematoma Associated with Angiographically Occult Arteriovenous Malformation -Case Report-
}

\author{
Hiroshi SAKaIda, Makoto Sakakura, Hiroshi TochIo, Kouji NaKaO, \\ Akira TANIGUCHI ${ }^{*}$ and Tadashi YABANA** \\ Departments of Neurosurgery, ${ }^{*}$ Neurology, and ${ }^{* *}$ Pathology, \\ Yamada Red Cross Hospital, Mie
}

\begin{abstract}
A 34-year-old female presented with a rare chronic encapsulated intracerebral hematoma associated with an angiographically occult arteriovenous malformation. A neovascularized fibrous capsule containing various stages of intracerebral hematoma formation was removed. These unusual entities mimic brain tumors or abscesses because of gradual growth and slowly progressive neurological deficits. Repeated bleeding or exudation from the capillaries of the capsule may allow expansion of the chronic encapsulated intracerebral hematoma.
\end{abstract}

Key words: chronic encapsulated intracerebral hematoma, arteriovenous malformation, fibrous capsule, ring enhancement

\section{Introduction}

Chronic encapsulated intracerebral hematomas may demonstrate fibrous capsules at surgery. ${ }^{1-7,9,11-16,18-}$ ${ }^{20,23)}$ However, such cases associated with arteriovenous malformation are very rare. ${ }^{5,16)}$ We report a 34-year-old female with chronic encapsulated intracerebral hematoma associated with an angiographically occult arteriovenous malformation, and discuss the etiology and mechanisms of encapsulation.

\section{Case Report}

A 34-year-old female had suffered from occasional migraine for 10 years, and had developed increasing severity of headache with nausea and vomiting since June, 1990. She was referred to our hospital on July 20, 1990, with a presumptive diagnosis of brain tumor based on computed tomography (CT) performed at a local hospital. She denied any history of head injury, epilepsy, hypertension, or coagulopathy.

Physical and neurological examination on admission revealed no abnormalities. All laboratory examinations were within normal ranges. Precontrast CT scans demonstrated a well-demarcated isodense mass in the left temporal lobe surrounded by a hyperdense zone. Postcontrast CT scans showed distinct ring enhancement, but no enhancement of the mass (Fig. 1). Magnetic resonance (MR) imaging detected heterogeneous signals in the round mass. The $T_{1}$-weighted image showed central iso- and peripheral high-intensity signals with thin rim enhancement using gadolinium-diethylenetriaminepenta-acetic acid (Gd-DTPA). The $\mathrm{T}_{2}$-weighted image showed the lesion as a marked high-intensity area separated from the surrounding edema by a low-intensity rim (Fig. 2). Cerebral angiograms revealed only mass effect.

On August 6, 1990, a left frontotemporal

Received November 27, 1992; Accepted February 1, 1993

Author's present address: H. Sakaida, M.D., Department of Neurosurgery, Faculty of Medicine, Mie University, Tsu, Japan. 

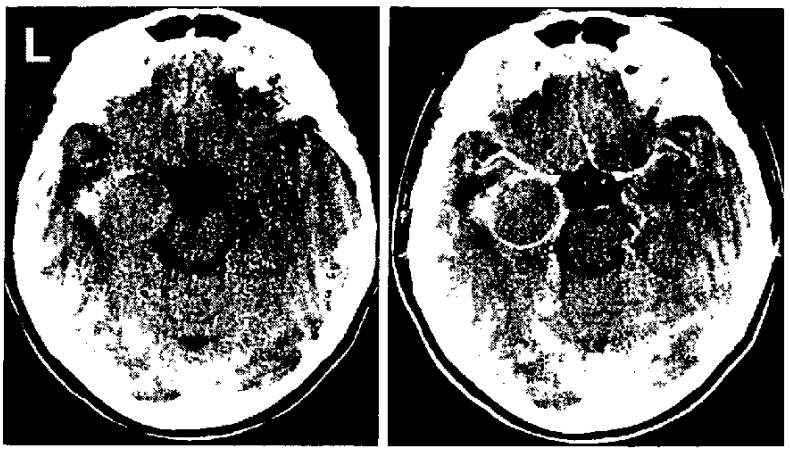

Fig. 1 left: Precontrast CT scan, showing a round isodense mass surrounded by a thin hyperdense zone in the left temporal lobe. right: Postcontrast CT scan, showing a nonenhanced isodense mass with distinct ring enhancement.

craniotomy was performed under a diagnosis of chronic intracerebral hematoma. A well-defined, tough capsule was found about $15 \mathrm{~mm}$ from the temporal cortex. Incision of the capsule revealed the liquid and solid components of the hematoma. The fibrous capsule was about $2 \mathrm{~mm}$ thick. A small red vein was noted in the medial pericapsular region, and a tiny vascular malformation was removed with a small amount of adjacent brain tissue.

Histological examination of the surgical samples revealed that the capsule consisted of abundant collagenous tissue on the outside and fibroblastic granulation tissue with rich neovascularity on the inside. Hemosiderin-laden macrophages were also present in the granulation tissue layer. The vascular

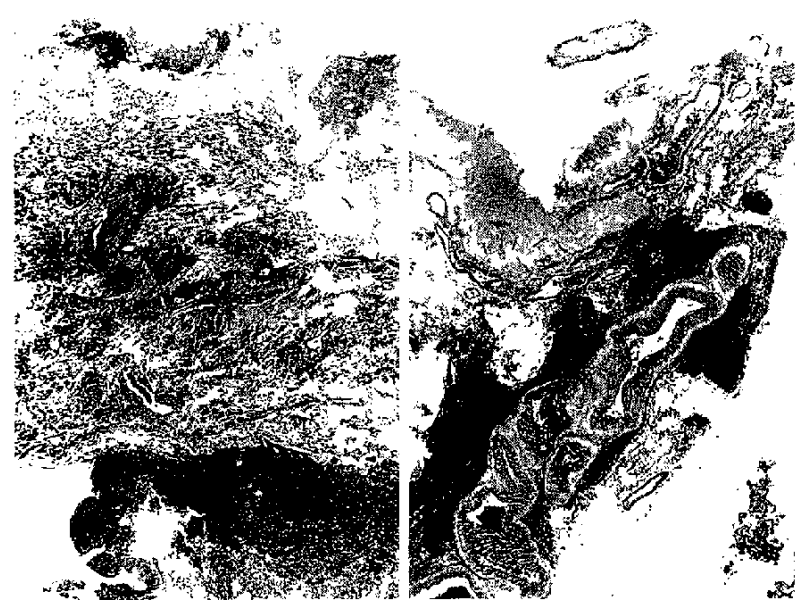

Fig. 3 left: Photomicrograph of the capsule, consisting of abundant collagenous tissue on the outside and fibroblastic granulation tissue, hemosiderin-laden macrophages, and rich neovascularity on the inside. right: Photomicrograph of the surgical specimen of the arteriovenous malformation. HE stain, $\times 30$.

anomaly was confirmed as an arteriovenous malformation (Fig. 3).

The postoperative course was satisfactory and she was discharged without neurological deficit on August 24, 1990.

\section{Discussion}

Hirsh et $a l .^{5)}$ first reported chronic encapsulated intracerebral hematoma in 1981, but two previous descriptions of this condition were reported. ${ }^{6.23)}$
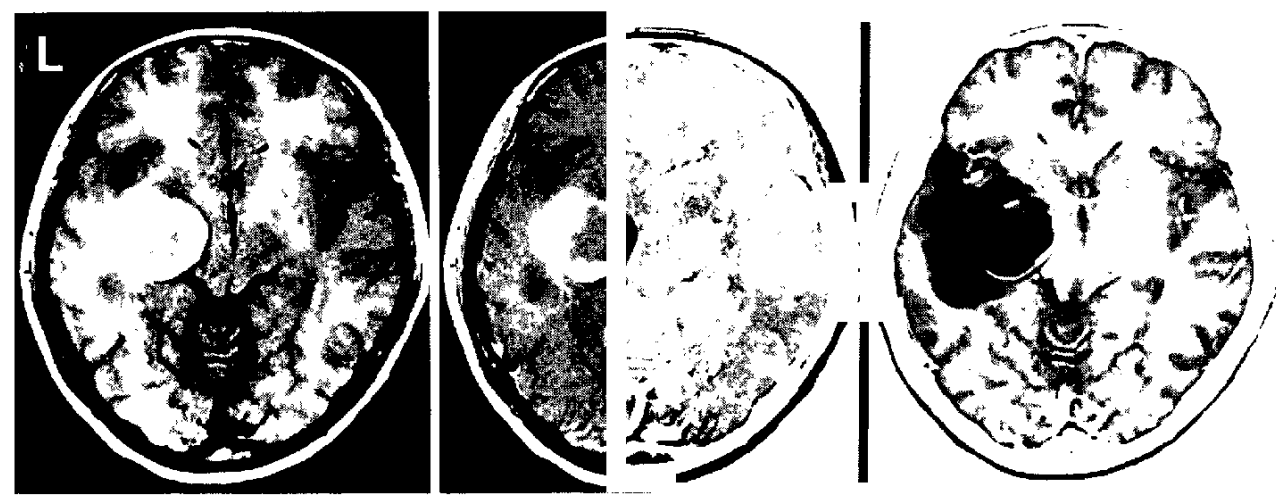

Fig. 2 left: $\mathrm{T}_{1}$-weighted MR image, showing a mixed high-intensity mass with a low-intensity boundary of the medial surface. center: $T_{1}$-weighted MR image with Gd-DTPA, showing rim enhancement with no enhancement of the mass. right: $\mathrm{T}_{2}$-weighted $\mathrm{MR}$ image, showing the markedly high-intensity content of the mass, emphasizing the peripheral low-intensity rim and perifocal edema. 
Table 1 Summary of 32 cases of chronic encapsulated intracerebral hematomas

\begin{tabular}{|c|c|c|c|c|c|c|c|c|}
\hline Author (Year) & Age & Sex & Side & Location & Signs and symptoms & $\begin{array}{c}\text { Dura- } \\
\text { tion }\end{array}$ & Etiology & $\begin{array}{l}\text { Out- } \\
\text { come* }\end{array}$ \\
\hline$\underset{(1977)^{23\}}}{\operatorname{Zimmerman}}$ et $a l$. & 25 & M & - & frontal & seizure & - & head trauma & - \\
\hline$\underset{(1978)^{6)}}{\text { Kawakami et al. }}$ & 31 & $\mathrm{~F}$ & middle & cerebellum & $\begin{array}{l}\text { headache, dizziness, } \\
\text { cerebellar signs }\end{array}$ & $8 \operatorname{mos}$ & unknown & GR \\
\hline \multirow[t]{2}{*}{$\begin{array}{l}\text { Hirsh et al. } \\
\qquad(1981)^{5)}\end{array}$} & 64 & $\mathbf{M}$ & $\mathrm{rt}$ & parieto-occipital & $\begin{array}{l}\text { It arm weakness, } \\
\text { lt homonymous hemianopsia }\end{array}$ & $2 \mathrm{mos}$ & AVM & $\mathrm{MD}$ \\
\hline & 72 & M & $\mathrm{rt}$ & parieto-occipital & It hemiparesis & - & AVM & MD \\
\hline$\underset{(1983)^{(9)}}{\text { Takahashi et al. }}$ & 55 & $\mathbf{F}$ & $\mathrm{rt}$ & parietotemporal & seizure & $17 \mathrm{yrs}$ & unknown & $\mathrm{MD}$ \\
\hline $\begin{array}{l}\text { Aoki and Mizuguchi } \\
(1984)^{1 !}\end{array}$ & $7 \mathrm{mos}$ & M & middle & cerebellum & $\begin{array}{l}\text { disturbance in holding } \\
\text { head steady }\end{array}$ & - & unknown & SD \\
\hline \multirow[t]{3}{*}{$\begin{array}{l}\text { Lin } e t a l . \\
\quad(1984)^{9)}\end{array}$} & 56 & M & it & frontoparietal & It hemiparesis, aphasia & $8 \mathrm{mos}$ & head trauma & MD \\
\hline & 44 & $\mathrm{~F}$ & $\mathrm{rt}$ & parietotemporal & seizure, It agnosia of body & $12 \operatorname{mos}$ & unknown & GR \\
\hline & 21 & $\mathbf{M}$ & It & frontotemporal & $\begin{array}{l}\text { headache, blurred vision, } \\
\text { lt abducens palsy }\end{array}$ & $2 \operatorname{mos}$ & unknown & GR \\
\hline$\underset{(1985)^{201}}{\text { Terada } a t}$ & 1 & $\mathrm{~F}$ & lt & temporal & $\begin{array}{l}\text { seizure, yomiting, } \\
\text { rt facial palsy }\end{array}$ & $2 \mathrm{wks}$ & unknown & GR \\
\hline \multirow[t]{2}{*}{$\begin{array}{l}\text { Masuzawa et al. } \\
(1985)^{12}\end{array}$} & 43 & $\mathbf{M}$ & $\mathrm{rt}$ & parietal & seizure, It hemiparesis & - & cavernoma & MD \\
\hline & 8 & $\mathbf{M}$ & $\mathrm{rt}$ & cerebellum & $\begin{array}{l}\text { headache, vomiting, } \\
\text { loss of consciousness }\end{array}$ & $3 \operatorname{mos}$ & unknown & GR \\
\hline $\begin{array}{l}\text { Aoki and Mizuguchi } \\
(1986)^{2)}\end{array}$ & 24 & $\mathrm{M}$ & $\mathrm{rt}$ & cerebellum & headache, cerebellar signs & 1 mo & unknown & $\mathrm{GR}$ \\
\hline \multirow{8}{*}{$\begin{array}{l}\text { Pozzati et al. } \\
(1986)^{16)}\end{array}$} & 66 & F & - & parieto-occipital & headache, papilledema & - & unknown & died \\
\hline & 11 & $\mathrm{M}$ & $\mathrm{rt}$ & frontoparietal & seizure & - & unknown & GR \\
\hline & 36 & $\mathrm{M}$ & $\mathrm{rt}$ & temporal & seizure & - & unknown & GR \\
\hline & 31 & F & - & frontal & seizure & - & unknown & GR \\
\hline & 62 & $F$ & lt & frontal & $\begin{array}{l}\text { frontal lobe syndrome, } \\
\text { papilledema }\end{array}$ & - & unknown & GR \\
\hline & 65 & $\mathrm{M}$ & - & frontal & headache & - & AVM & GR \\
\hline & 44 & M & - & parieto-occipital & seizure, It hemiparesis & - & unknown & GR \\
\hline & 57 & $\mathbf{M}$ & - & frontoparietal & It hemiparesis & - & unknown & died \\
\hline $\begin{array}{l}\text { Steiger et al. } \\
\quad(1987)^{18)}\end{array}$ & 45 & $\mathbf{M}$ & - & frontal & hemiparesis, papilledema & - & cavernoma & - \\
\hline Chan and Tse & 20 & $\mathbf{M}$ & lt & parietotemporal & headache, diplopia & $3 \operatorname{mos}$ & UCVM & GR \\
\hline $\begin{array}{l}\text { Matsumoto et al. } \\
\qquad(1988)^{1,3)}\end{array}$ & 60 & $\mathrm{~F}$ & lt & occipital & $\begin{array}{l}\text { headache, } \\
\text { rt homonymous hemianopsia }\end{array}$ & $2 \operatorname{mos}$ & $\begin{array}{l}\text { metastatic } \\
\text { adenocarcinoma }\end{array}$ & GR \\
\hline \multirow[t]{2}{*}{$\begin{array}{l}\text { Fiumara et } a l . \\
(1989)^{4)}\end{array}$} & 33 & $\mathrm{M}$ & It & parietal & $\begin{array}{l}\text { seizure, dysphasia, } \\
\text { dyslexia, dysgraphia }\end{array}$ & $4 \operatorname{mos}$ & unknown & $\mathrm{MD}$ \\
\hline & 64 & M & $\mathrm{rt}$ & frontal & frontal lobe syndrome & 3 mos & unknown & $\mathrm{MD}$ \\
\hline \multirow[t]{2}{*}{$\underset{(1990)^{(4)}}{\operatorname{Monma} \text { et }}$} & 29 & $\mathbf{F}$ & $\mathrm{rt}$ & parietal & seizure & $21 \mathrm{yrs}$ & cavernoma & MD \\
\hline & 32 & $\mathrm{M}$ & $\mathrm{rt}$ & frontal & headache & $1 \mathrm{wk}$ & cavernoma & GR \\
\hline$\underset{(1990)^{7}}{\text { Kumabe et al. }}$ & 29 & M & $\mathrm{rt}$ & basal ganglia & $\begin{array}{l}\text { headache, } \\
\text { lt lower quadrantoanopsia }\end{array}$ & $2 \mathrm{mos}$ & venous angioma & GR \\
\hline$\underset{(1990)^{15)}}{\operatorname{Murakami}}$ et al. & 14 & $\mathbf{M}$ & rt & frontal & headache, nausea, vomiting & $2 \mathrm{wks}$ & cavernoma & GR \\
\hline $\begin{array}{l}\text { Mabuchi et al. } \\
\quad(1991)^{113}\end{array}$ & 43 & $\mathrm{~F}$ & It & parietal & $\begin{array}{l}\text { headache, nausea, } \\
\text { rt hemiparesis }\end{array}$ & $19 \operatorname{mos}$ & cavernoma & GR \\
\hline Present case & 34 & $\mathbf{F}$ & It & temporal & headache, nausea, vomiting & 10 yrs & AVM & $\mathrm{GR}$ \\
\hline
\end{tabular}

${ }^{*}$ Glasgow Outcome Scale; GR: good recovery, MD: moderate disability, SD: severe disability. AVM: arteriovenous malformation, UCVM: unclassified vascular malformation.

Chronic encapsulated hematomas occur extracranially and in many other locations of the body. ${ }^{17)}$

Table 1 lists the 31 reported cases of chronic encapsulated intracerebral hematomas with surgically proven fibrous capsules. ${ }^{1-7,9,11-16,18-20,23)}$ Two cases strongly resembling this entity were excluded because no surgical confirmation of the fibrous capsules was reported. ${ }^{21,22)}$ The neurological signs and symptoms 
are not specific as with space-occupying lesions, but slow progression or increased frequency occurred in most patients.

The etiology of the hemorrhage was detected in 15 cases: 12 were caused by vascular malformations including six cavernomas, ${ }^{11,12,14,15,18)}$ four arteriovenous malformations, ${ }^{5,16)}$ one venous malformation, ${ }^{7)}$ and one unclassified vascular malformation. ${ }^{3)}$ The other etiologies were two head injuries ${ }^{9,23)}$ and a metastatic adenocarcinoma. ${ }^{13)}$ Our case is unique in that the vascular malformation was a true arteriovenous malformation histologically. Previous possible cases ${ }^{5,16)}$ did not provide sufficiently detailed information, and many cases are unknown. The incidence of this lesion associated with arteriovenous malformation cannot be estimated and it is unclear why encapsulated hematoma in this setting is rare.

Chronic encapsulated intracerebral hematomas do not develop from hypertensive intracerebral hemorrhages. Postcontrast CT demonstrates resolving intracerebral hematomas with a ring of enhancement. Laster $e t a l{ }^{8)}$ reported that early ring formation was related to blood-brain-barrier breakdown and later enhancement to vascular granulation tissue based on histological examination. The final stage was the healed phase of hematoma resorption, during which no ring enhancement occurred. Therefore, another cause of the capsule formation should be considered.

The tough, fibrous capsule of chronic encapsulated intracerebral hematoma is similar to the outer membrane of chronic subdural hematoma, ${ }^{2)}$ with abundant fibrous connective tissue with rich neovascularity. Hirsh et al. ${ }^{5)}$ suggested that the capsule originated from fibroblasts related to the abnormal vessels of an occult vascular malformation. Lobato et $a .^{10)}$ found that over half of 262 angiographically occult intracranial vascular malformations had caused macroscopically visible intraparenchymal hematomas (either recent or old) and/or intraventricular or subarachnoid hemorrhage. Most cases had repeated ictal episodes for variable periods before the causative lesion was identified and removed. Therefore, repeated bleeding may encourage capsule formation with the rich neovascularity caused by the fibroblasts from the vascular malformation. A common hypothesis is that repeated bleeding or exudation from the capillaries of the capsule allows expansion of the chronic encapsulated intracerebral hematoma.

The 31 reviewed cases all had fibrous capsules, and most hematomas were totally removed. The postoperative courses of most patients were satisfactory. Two deaths were reported but details are unclear. ${ }^{16)}$ The necessity for total removal of the hematoma with the capsule is still uncertain. Therefore, further investigation of the optimum treatment is necessary.

\section{References}

1) Aoki N, Mizuguchi K: Chronic encapsulated hematoma intracerebellar in infancy: Case report. Neurosurgery 14: 594-597, 1984

2) Aoki N, Mizuguchi K: Expanding intracerebellar hematoma: A possible clinicopathological entity. Neurosurgery 18: 94-96, 1986

3) Chan ST, Tse CHC: Chronic encapsulated intracerebral hematoma in a young Chinese adult: Case report. Neurosurgery 20: 639-642, 1987

4) Fiumara E, Gambacorta M, D'Angelo V, Corona C: Chronic encapsulated intracerebral haematoma: Pathogenetic and diagnostic considerations. J Neurol Neurosurg Psychiatry 52: 1296-1299, 1989

5) Hirsh LF, Spector HB, Bogdanoff BM: Chronic encapsulated intracerebral hematoma. Neurosurgery 9: 169-172, 1981

6) Kawakami Y, Nakao Y, Tabuchi K, Nosaka Y, Ohmoto T: Bilateral intracerebellar calcification associated with cerebellar hematoma: Case report. $J$ Neurosurg 49: 744-748, 1978

7) Kumabe T, Kayama T, Sakurai Y, Ogasawara K, Niizuma $H$, Wada $T$, Namiki $T$ : Encapsulated chronic intracerebral hematoma caused by venous angioma at basal ganglia. No Shinkei Geka 18: 735739, 1990 (in Japanese)

8) Laster DW, Moody DM, Ball MR: Resolving intracerebral hematoma: Alteration of the "ring sign" with steroids. $A J R$ 130: 935-939, 1978

9) Lin SZ, Shih CJ, Wang YC, Tsai SH: Intracerebral hematoma simulating a new growth. Surg Neurol 21: 459-464, 1984

10) Lobato RD, Perez C, Rivas JJ, Cordobes F: Clinical, radiological, and pathological spectrum of angiographically occult intracranial vascular malformations: Analysis of 21 cases and review of the literature. $J$ Neurosurg 68: 518-531, 1988

11) Mabuchi S, Kamiyama $\mathrm{H}$, Iwasaki $\mathrm{Y}$, Abe $\mathrm{H}$ : Multilocular encapsulated intracerebral hematoma: A case report. No Shinkei Geka 19: 677-679, 1991 (in Japanese)

12) Masuzawa $T$, Saito $K$, Shimabukuro $H$, Iwasa $H$, Sato F: Chronic encapsulated hematomas in the brain. Acta Neuropathol (Berl) 66: 24-28, 1985

13) Matsumoto $K$, Asari $S$, Nishimoto A, Namba $S$ : Chronic encapsulated intracerebral hematoma due to metastatic brain tumor: A case report. No Shinkei Geka 16: 327-331, 1988 (in Japanese)

14) Monma $S$, Ohno $K$, Hata $H$, Komatsu $K$, Ichimura $\mathrm{K}$, Hirakawa $\mathrm{K}$ : Cavernous angioma with encapsulated intracerebral hematoma: Report of two cases. Surg Neurol 34: 245-249, 1990

15) Murakami S, Sotsu M, Morooka S, Suzuki T: 
Chronic encapsulated intracerebral hematoma associated with cavernous angioma: A case report. Neurosurgery 26: 700-702, 1990

16) Pozzati E, Giuliani G, Gaist G, Piazza G, Vergoni G: Chronic expanding intracerebral hematoma. $J$ Neurosurg 65: 611-614, 1986

17) Reid JD, Kommareddi S, Lankerani M, Park MC: Chronic expanding hematomas: A clinicopathologic entity. JAMA 244: 2441-2442, 1980

18) Steiger HJ, Markwalder TM, Reulen HJ: Clinicopathological relations of cerebral cavernous angiomas: Observations in eleven cases. Neurosurgery 21: 879-884, 1987

19) Takahashi N, Kikuchi H, Kobayashi K, Karasawa J: Multilocular encapsulated intracerebral hematoma. No Shinkei Geka 11: 739-743, 1983 (in Japanese)

20) Terada $T$, Okuno $T$, Moriwaki H, Nakai E, Komai
N: Chronic encapsulated intracerebral hematoma during infancy: Case report. Neurosurgery 16: $833-835$, 1985

21) Weisberg LA, Stazio A, Elliott D: The chronic expanding intracerebral hematoma. Comput Med Imaging Graph 14: 61-65, 1990

22) Yashon D, Kosnik EJ: Chronic intracerebral hematoma. Neurosurgery 2: 103-106, 1978

23) Zimmerman RD, Leeds NE, Naidich TP: Ring blush associated with intracerebral hematoma. Radiology 122: $707-711,1977$

Address reprint requests to: H. Sakaida, M.D., Department of Neurosurgery, Faculty of Medicine, Mie University, 2-174 Edobashi, Tsu 514, Japan. 